diseased coronary arteries ( $>50 \%$ stenosis) was used to assess the extent of CAD.

Results HuR mRNA expression was significantly increased in patients with CAD (both stable and AMI) compared to controls $(p=0.039)$. Subgroup analysis revealed that STEMI patients $(n=107)$ had increased levels of HuR expression compared to NSTEMI $(n=49, p=0.03)$. Among patients with stable CAD $(n=133)$, high HuR expression was independently associated with the number of diseased coronary arteries $(\mathrm{OR}=1.35$ for $1-\mathrm{SD}$ increase in $\mathrm{HuR}, 95 \% \mathrm{CI} 1.07-1.72$, $\mathrm{p}=0.012)$, as well as with reduced ejection fraction $(\mathrm{EF}<45 \%$, $\mathrm{OR}=1.32$ per 1 -SD increase, 95\% CI 1.05-1.85, $\mathrm{p}=0.024)$. Among individuals without $\mathrm{CVD}$, high $\mathrm{HuR}$ was associated with lower HDL levels (adjusted beta $=-5.2 \mathrm{mg} / \mathrm{dl}$ for highest versus lowest quartile, $\mathrm{p}=0.03$ ) and higher diastolic blood pressure (adjusted beta $=3.6 \mathrm{mmHg}, \mathrm{p}=0.007$ ), while, after adjustment for traditional cardiovascular risk factors, HuR levels in individuals without CVD were independently associated with increased IMT in the common carotid artery (mean increase $6.2 \%$ for highest versus lowest quartile, $p=0.019$ ).

Conclusion $\mathrm{HuR}$ expression is associated with early subclinical arterial disease in individuals without clinically overt CVD and with the presence and severity of cardiac and vascular dysfunction in patients with clinically overt CAD. These findings imply a clinical role of the HuR pathway in cardiovascular disease and warrant further investigation.

Conflict of Interest none

\section{QUALITY OF CONFLICTS OF INTEREST DECLARATIONS IN A CONFERENCE SETTING - ARE AUDIENCES GIVEN A CHANCE TO ADEQUATELY ASSESS BIAS?}

${ }^{1}$ William Crawford*, 'C. Fielder Camm, Ishika Prachee, ${ }^{2}$ Matthew Ginks. 'University of Oxford; ${ }^{2}$ Oxford University Hospitals

\subsection{6/heartjnl-2019-BCS.112}

Introduction Potential conflicts of interest (COIs) are common. Interaction with industry is often required to develop cardiology research. However, it has been suggested that COIs may affect professional opinion objectivity. As a result, appropriate COI declarations are required when presenting research.
Methods Recorded presentations from the British Cardiovascular Society annual conference 2018 were reviewed. Presentations were excluded if they were panel discussions, chairperson remarks or motivational talks, or slides were not available. A 3-point quality score was developed to assess declarations; 1) was a COI slide displayed for a minimum sufficient time $(250 \mathrm{~ms} /$ conflict $), 2)$ the presence of a verbal COI explanation, and 3$)$ use of a font size comparable $(<10 \%$ smaller) to the BCS default. Presentations were given a score of $0-3$ based on these criteria and compared against the presenter's H-index as a surrogate marker of seniority.

Results A total of 84 presentations were available for review; 70 met the inclusion criteria, and 48 (69\%) contained a COI statement. Of those with a statement, the median number declared was 0 (IQR 0 to 1 ); while 12 (25\%) declared at least one COI. Of those presenters with at least one COI, 5 (41\%) did not offer a verbal explanation; 5 (41\%) used a font size smaller than the default; and conflicts were displayed for a median time of $1 \mathrm{~s}$ per conflict (IQR 0.8-3.0s). Three $(25 \%)$ presenters with a potential COI declared it with a quality score of 3 , while $1(8 \%)$ presenter scored 1 . No presenters scored 0 (see figure). Linear regression models showed a presenter's H-index was predictive of the number of conflicts reports (OR 1.1 95\% CI [1.05 to 1.15$], \mathrm{p}<0.001, \mathrm{n}=45$ ), but

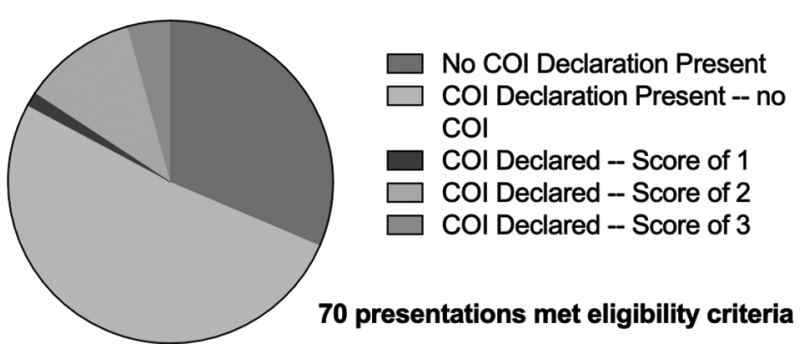

Abstract 115 Figure $1 \mathrm{COI}$ declaration status of all presentations meeting inclusion criteria.

Of the 70 presentations assessed, $22(31.4 \%)$ did not contain a COI declaration. Thirty-six (51.4\%) contained a COI declaration, but had no potential conflict to declare. Twelve $(17.1 \%)$ presentations contained a COI declaration, and declared at least one potential conflict. Using our devised declaration quality score, three $(4.3 \%)$ had a score of three, eight $(11.4 \%)$ had a score of two, and one (1.4\%) had a score of 1. a)

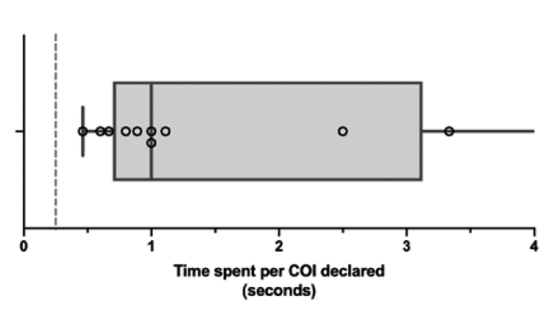

b)

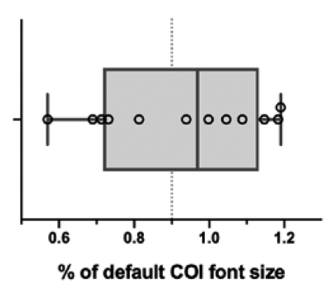

c)

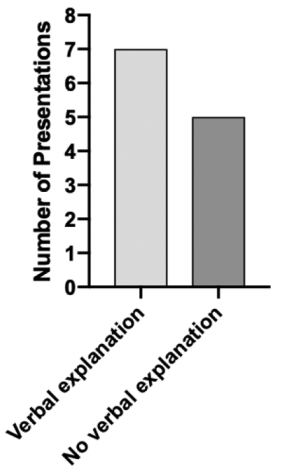

Abstract 115 Figure 2 Scoring parameters for presentations with potential conflicts of interest $(n=12)$.

a) All presentations with potential conflicts spent an adequate amount of time per declaration on their declaration slide (median=1.0s, IQR 0.8-3.0s). b) Five presentations $(41 \%)$ with a potential conflict declared this with a font size $<90 \%$ of the default BCS COI declaration font size. c) Five (41\%) presentations with a potential conflict did not offer a verbal explanation of their conflict. 
not time spent per COI (OR 0.89, 95\% [0.68 to 1.16], $\mathrm{p}=0.38, \mathrm{n}=11$ ). An ordinal regression model did not show an association between presenter's $\mathrm{H}$-index and total quality score (OR $0.94,95 \%$ CI [0.87 to 1.01$], \mathrm{p}=0.11, \mathrm{n}=11$ )

Conclusion A large minority of presenters did not display a COI statement. Those COI statements that were present were of variable quality. Many were displayed for insufficient time, lacked any verbal explanation or used a small font. These factors may limit the ability of audience members to understand the potential conflicts. Seniority of presenter correlated with number of conflicts but not quality of the COI statement. Strategies should be implemented to ensure all presenters outline whether they have any potential COI in a standardised manner.

Conflict of Interest None

\section{ANTIHYPERTENSIVE DRUG CONCORDANCE IN PATIENTS WITH APPARENT RESISTANT HYPERTENSION: A TERTIARY HYPERTENSION REFERRAL CENTRE EXPERIENCE}

Tarek Antonios*. St George's, University of London, London

10.1136/heartjnl-2019-BCS.113

Introduction Individuals with resistant hypertension have a $50 \%$ greater risk of cardiovascular events (death, myocardial infarction, heart failure, stroke or chronic kidney disease) compared to those with well-controlled hypertension. Nonadherence to antihypertensive medication is a major cause of uncontrolled hypertension, with rates reported as high as $84 \%$. The aim of this study was to assess the prevalence of non-adherence in hypertensive individuals referred to a tertiary hypertension centre in South of London (Blood Pressure Unit, St George's University Hospital NHS foundation Trust).

Methods After the optimisation of drug regimens and the exclusion of white coat and secondary hypertension, drug adherence was assessed using liquid chromatography-mass spectrometry (LC-MS) analysis of urine samples in 124 individuals who met the criteria for resistant hypertension (uncontrolled hypertension despite concurrently taking at least 3 antihypertensive drugs of different classes including a diuretic or hypertension controlled on 4 drugs). All included subjects confirmed adherence to their prescribed antihypertensive medication on several occasions, whilst individuals who admitted to missing their drugs were excluded from the study.

Results Non-adherence was found in 71 individuals (57.3\%). Of these $45(63.4 \%)$ had partial non-adherence and 26 $(36.6 \%)$ had complete non-adherence. Non-adherent individuals were significantly younger in age, had a shorter history of hypertension, more likely to be a female (OR: 3.07; 95\% CI: 1.45-6.47; $\mathrm{p}=0.003$ ) and have depression (OR: 11.66; 95\% CI 1.47-92.19; $\mathrm{p}=0.004)$, and less likely to have concomitant cardiovascular disease (OR: $0.42 \quad 95 \%$ CI $0.19-0.92$; $\mathrm{p}=0.028$ ), type 2 diabetes mellitus (OR: 0.40 ; 95\% CI: $0.19-$ $0.83 ; \mathrm{p}=0.013$ ), chronic kidney disease (OR: 0.34; 95\% CI $0.12-0.92 ; \mathrm{p}=0.028$ ) and retinopathy (OR: $0.14 ; 95 \% \mathrm{CI}$ $0.04-0.51 ; \mathrm{p}=0.001)$. Low levels of adherence were observed more for individuals on diuretics.

Conclusion In individuals with apparent resistant hypertension, poor adherence to antihypertensive therapy is very common and was observed in $57 \%$ of individuals despite their insistence of their treatment adherence in the preceding days. The assessment of adherence at an early stage in individuals with apparent resistant hypertension is essential for tailoring treatment strategies and for saving vital healthcare resources.

Conflict of Interest None

\section{CAN NON-MODIFIABLE RISK FACTORS OF AGE AND SEX BE USED TO DETERMINE WHICH PATIENTS WITH CHEST PAIN WE INVESTIGATE WITH CT CORONARY ANGIOGRAPHY (CTCA) AS AN INITIAL TEST}

${ }^{1}$ Ali Rauf*, ${ }^{2}$ Shazia Arshad, ${ }^{3}$ Sarah Denny, ${ }^{3}$ Floyd Pierres, ${ }^{3}$ Nikolaos Papamichail, ${ }^{4}$ Antonis Pavlidis, ${ }^{3}$ Khaled Alfakih. ' University College London; ${ }^{2}$ The Grove Medical Practice; ${ }^{3}$ Lewisham and Greenwich NHS Trust; ${ }^{4}$ Guy's and St. Thomas' NHS Foundation Trust

\subsection{6/heartjnl-2019-BCS.114}

Introduction The UK NICE guidelines for the investigation of chest pain were updated in 2016 to recommend CTCA as the initial test for all patients with stable chest pain. This was in comparison to the 2010 guidelines on stable chest pain which recommended that patients with mild and moderate pre-test probability (PTP) should be investigated non-invasively with cardiac CT and functional imaging tests respectively whilst patients with high PTP should be referred directly to invasive coronary angiography (ICA). The removal of PTP in the updated guidelines was in view of it overestimating prevalence of coronary artery disease (CAD).

Purpose We wanted to assess whether non-modifiable risk factors of age and sex can be used to better predict which patients with new onset chest pain should be investigated by a non-invasive test such as CTCA and whether there is still a role for direct referral for ICA

Method We examined our catheter lab database for patients undergoing ICA over a 12 month period for either new onset stable chest pain or acute coronary syndrome (ACS) who had not undergone any initial non-invasive test. We documented age and sex for all patients, and calculated percentage yield of severe CAD in each of the subgroups.

Results In total, 330 patients with stable chest pain and 250 with ACS were identified. The prevalence of severe CAD in these cohorts, grouped according to age and sex, are shown in table 1. Numbers of females under the age of 55 were lowest as might be expected, and this also corresponded with low \% yield of severe CAD in both the stable (14\%) and ACS (21\%) group. In the stable group, prevalence of severe $\mathrm{CAD}$ was relatively low in all other groups, and highest in males aged over 55 at $40 \%$. Percentage yield was above $50 \%$ in all males with ACS, and all females above the age of 55 .

Conclusion Our data demonstrates that in the patients with stable symptoms, who were referred directly for ICA and therefore had presumed high PTP, there was low prevalence of severe CAD particularly in young females. This would support NICE guidance to investigate these patients non-invasively. There may still be a role for investigating older men with ICA given the relatively higher prevalence. In patients presenting with ACS, our data suggests that there may also be a role for investigating young women by non-invasive means in the first instance.

Conflict of Interest None 